\title{
BOS releases teledentistry guidance
}

The British Orthodontic Society (BOS) has released its guidance on teledentistry and remote interactions in orthodontic care.

Further to statements from both the GDC and the CQC in 2021, the BOS have produced this guidance to help orthodontic providers and their teams better understand the scope of teledentistry services and technologies as part of orthodontic care, as well as the associated issues. In addition, it will direct teams to the current regulatory frameworks, resources and highlight best operational practice.

Teledentistry is a term which covers a range of technologies and operational practices.

These include various communications via interactive, two-way audio or video as well as indirect, synchronous communications, in which a patient's information (such as questions, requests, photographs, videos) is exchanged with a dental professional for review. This may be via messaging platforms or dedicated hardware and applications.

Teledentistry can enhance patient care, assist in achieving agreed treatment outcomes, and strengthen the relationship between clinician and patient. Potential benefits of integrating teledentistry technologies and procedures into orthodontic care include greater accessibility, better patient engagement and experience, reduced physical appointments - ideal from a COVID-19 perspective as well as reduced carbon footprint, and above all, increased treatment efficiency.

In accordance with the GDC Scope of Practice, all diagnostic and prescriptive decisions must be made by the treating orthodontist/dentist who have adequate training and skills. The direct involvement of an appropriately trained and registered orthodontist/dentist is essential for the monitoring of orthodontic care. Patients undergoing treatment must be made aware of the name of the clinician responsible for their care and be able to make direct contact with this clinician as well as be able to arrange appropriate face-to-face appointments when required. Patients should be informed that clear aligner treatment, even for cosmetic purposes, is not a simple process but is a medical procedure using a medical device. Treatment should only be undertaken with the direct guidance and ongoing supervision of a named orthodontist or suitably trained dentist.
The BOS still has significant concerns surrounding the appropriate examination, diagnosis and consent process for 'DIY orthodontic' systems as well as ongoing supervision and the nature of the relationship between patient and treating clinician.

BOS Director of External Relations, Anjli Patel commented: 'If used responsibly, digital technologies and associated tools or applications have potential to improve professional care and enhance both patient outcomes and convenience, adding a potentially convenient way for clinicians to engage with current or prospective patients. It can enhance patient care, assist in achieving agreed treatment outcomes, and strengthen the relationship between clinician and patient. However, it should never be used to alter clinical practice in such a way that patient safety, valid consent or treatment planning and outcomes are compromised.'

To view the guidance visit https://view. publitas.com/british-orthodontic-society/ guidance-on-teledentistry-and-remoteinteractions-in-orthodonticcarefinal-v5awamended/ page/1.

\section{NHS dentistry: little urgency from government to solve access crisis}

The British Dental Association has mourned the government's lack of urgency on reforming NHS dentistry, following a major debate in the House of Commons.

New data published shows 40 million NHS appointments - the equivalent of a whole year's worth of care in pre-COVID times - have been lost since lockdown.
Ministers have declined to set a deadline to reform the widely discredited NHS dental contract - which is fuelling an exodus from the service - or guarantee adequate funding is put in place to underpin the rebuild of the service. Despite a recent pledge of $£ 50 \mathrm{~m}$ to provide 350,000 appointments by 1 April, the service has faced unprecedented cuts over the last decade and would require an additional $£ 880 \mathrm{~m}$ simply to restore levels of resources to 2010 levels.

Despite recognising dentistry as the 'Cinderella Service', Health Minister Maria Caulfield failed to set a date on breaking from the current system, despite being pressed by MPs on both sides of the House.

The BDA has discounted 'quick fix' solutions to import dentists from overseas. There are over 42,000 dentists currently on the General Dental Council's official register, up 5\% of 2018 levels, just not enough willing to work under the current NHS system. The union has stressed the imperative to develop a service that encourages UK graduates to view the NHS as a place to build a career.

BDA Chair Eddie Crouch said: 'England has lost a year's worth of dentistry, but the government has yet to show it recognises the urgency here.

'NHS dentistry will keep haemorrhaging talent and millions will continue going without needed care until Ministers grasp the nettle.

'This service was in crisis even before COVID-19 struck, yet officials are still unwilling to call time on a failed NHS contract or reverse a decade of savage cuts.

'Calls to import staff from overseas would be just painting over the cracks. The problem isn't the number of dentists we have, we just don't have enough willing to work under a broken system. We need real change, not more quick fixes.' 